ARTICLE

\title{
Dynamic co-catalysis of Au single atoms and nanoporous Au for methane pyrolysis
}

Wei Xi ${ }^{1,3}$, Kai Wang (1) ${ }^{1,3}$, Yongli Shen ${ }^{1,3}$, Mengke Ge ${ }^{1}$, Ziliang Deng (i) ${ }^{1}$, Yunfeng Zhao ${ }^{1}$, Qiue Cao ${ }^{2}$, Yi Ding (i) ${ }^{1 凶}$, Guangzhi Hu (10 ${ }^{2 \times} \&$ Jun Luo (i) ${ }^{1 \times}$

Nanocatalysts and single-atom catalysts are both vital for heterogeneous catalysis. They are recognized as two different categories of catalysts. Nevertheless, recent theoretical works have indicated that Au nanoparticles/clusters release Au single atoms in $\mathrm{CO}$ oxidation, and they co-catalyze the oxidation. However, to date, neither experimental evidence for the cocatalysis nor direct observations on any heterogeneous catalysis process of single-atom catalysts are reported. Here, the dynamic process of nanoporous Au to catalyze methane pyrolysis is monitored by in situ transmission electron microscopy with high spatial-temporal resolutions. It demonstrates that nanoporous Au surfaces partially disintegrate, releasing $\mathrm{Au}$ single atoms. As demonstrated by DFT calculation, the single atoms could co-catalyze the reaction with nanoporous $\mathrm{Au}$. Moreover, the single atoms dynamically aggregate into nanoparticles, which re-disintegrate back to single atoms. This work manifests that under certain conditions, the heterogeneous catalysis processes of nanocatalysts and single-atom catalysts are not independent, where their dynamic co-catalysis exists.

\footnotetext{
${ }^{1}$ Center for Electron Microscopy and Tianjin Key Lab of Advanced Functional Porous Materials, Institute for New Energy Materials \& Low-Carbon Technologies, School of Materials Science and Engineering, Tianjin University of Technology, 300384 Tianjin, China. ${ }^{2}$ School of Chemical Science and Technology, Key Laboratory of Medicinal Chemistry for Nature Resource, Ministry of Education, Yunnan University, 650091 Kunming, China. ${ }^{3}$ These authors contributed equally: Wei Xi, Kai Wang, Yongli Shen. ${ }^{凶}$ email: yding@tjut.edu.cn; guangzhihu@ynu.edu.cn; jluo@tjut.edu.cn
} 
$\mathrm{H}$ eterogeneous catalysis is a crucial process in industries of chemicals, energy and environmental protection and their related fundamental researches, such as hydrogenation ${ }^{1-3}$, $\mathrm{CO}$ oxidation ${ }^{4-8}$, and $\mathrm{CH}_{4}$ activation and use $\mathrm{e}^{9-16}$. In its currently increasing development, nanocatalysts ${ }^{1-3,7,9-20}$ and single-atom catalysts (SACs) ${ }^{4,8,21-28}$ are two different categories of vital heterogeneous catalysts. The two categories have exhibited excellent performances and complementary functions, and their catalytic processes/mechanisms are generally considered to be different from each other ${ }^{24,25,28}$. Nevertheless, two recent works ${ }^{5,6}$ use theoretical calculations to indicate that during the $\mathrm{CO}$ oxidation catalyzed by $\mathrm{Au}$ nanoparticles/clusters, Au single atoms (SAs) can break away from the nanoparticles/clusters, and the SAs and the nanoparticles/clusters co-catalyze the CO oxidation. These results give rise to an inspiration that the gap between the heterogeneous catalytic processes of nanocatalysts and SACs would be bridged by the co-catalysis. Significantly, this inspiration is indirectly supported by experimental observations that metal nanoclusters or substrates can release metal SAs and they co-catalyze the growth of carbon nanostructures in all-solid-phase catalysis ${ }^{29-31}$. However, up to now, no experimental evidence has been reported for the emergence of SACs in any heterogeneous catalysis process of nanocatalysts. Meanwhile, even though the dynamic processes of metal SAs to catalyze the growth of carbon nanostructures in all-solid-phase catalysis have been impressively demonstrated $^{27,29-31}$, no published works in the literature give direct observations on any heterogeneous catalysis process of SACs. Thus, it remains elusive whether the co-catalysis exists in any heterogeneous catalysis process initiated by either of nanocatalysts and SACs.

Among heterogeneous catalysis reactions, the methane $\left(\mathrm{CH}_{4}\right)$ pyrolysis is a fully green single-step technology for producing nanocarbon and hydrogen $\left(\mathrm{H}_{2}\right)$, the most environmentally friendly energy carrier ${ }^{14-16}$. It is also important for mitigating the environmental challenge associated with $\mathrm{CH}_{4}$ emission ${ }^{12,13,15,16}$. Meanwhile, $\mathrm{Au}$ nanocatalysts and their derived catalysts have exhibited excellent catalytic activities for $\mathrm{CH}_{4}$ activation and use $^{9,10,14}$. As a new type of Au nanocatalyst with threedimensional (3D) bicontinuous porous structure ${ }^{32-38}$, nanoporous gold (NPG) has shown high performances in heterogeneous catalysis ${ }^{32,36,37}$. Its unique structure has large specific surface area, low density, and high permeability, which are all advantageous for heterogeneously catalytic reactions ${ }^{32-38}$. Therefore, we here use NPG to catalyze the $\mathrm{CH}_{4}$ pyrolysis and characterize its dynamic catalysis process by conventional and in situ transmission electron microscopy (TEM) with high spatial and temporal resolutions. The characterizations reveal that some of the NPG surfaces continuously disintegrate, from which Au SAs are released. The Au SAs and NPG co-catalyze the $\mathrm{CH}_{4}$ pyrolysis to produce amorphous carbon and $\mathrm{H}_{2}$. Moreover, during the catalysis process of the Au SAs, some SAs dynamically aggregate into $\mathrm{Au}$ nanoparticles, and the nanoparticles dynamically redisintegrate back to SAs. The nanoparticles and SAs also cocatalyze the $\mathrm{CH}_{4}$ pyrolysis, which is confirmed by our theoretical calculations. This work gives solid experimental evidence that under certain conditions of heterogeneous catalysis, SAs are released by nanocatalysts and then participate in catalytic reactions originally initiated by nanocatalysts, and vice versa. These results can help to bridge the gaps between the catalysis processes of nanocatalysts and SACs and provide new inspirations for designing the catalysts.

\section{Results}

Characterization of NPG before and after ex situ $\mathrm{CH}_{4}$ pyrolysis. Ex situ catalytic $\mathrm{CH}_{4}$ pyrolysis reaction was performed using NPG as the catalyst at $580^{\circ} \mathrm{C}$ in a system of direct-current plasma-enhanced chemical vapor deposition (dc-PECVD). The flow rates of $\mathrm{CH}_{4}, \mathrm{Ar}$, and $\mathrm{H}_{2}$ were 50,200 , and $20 \mathrm{sccm}(\mathrm{sccm}$ is standard cubic centimeters per minute), respectively, and their chamber pressure was 5 mbar (see "Methods" for more details; it should be noted that this dc-PECVD system is independent from and outside TEM). The structures of NPG before and after the ex situ reaction were investigated firstly by conventional scanning electron microscopy (SEM) and TEM techniques including highangle annular dark field (HAADF) imaging of scanning TEM (STEM), as shown in Fig. 1 and Supplementary Figs. 1-4.

The NPG samples were prepared by dealloying, and they possess bicontinuous pore channels and ligaments ${ }^{32-38}$. For

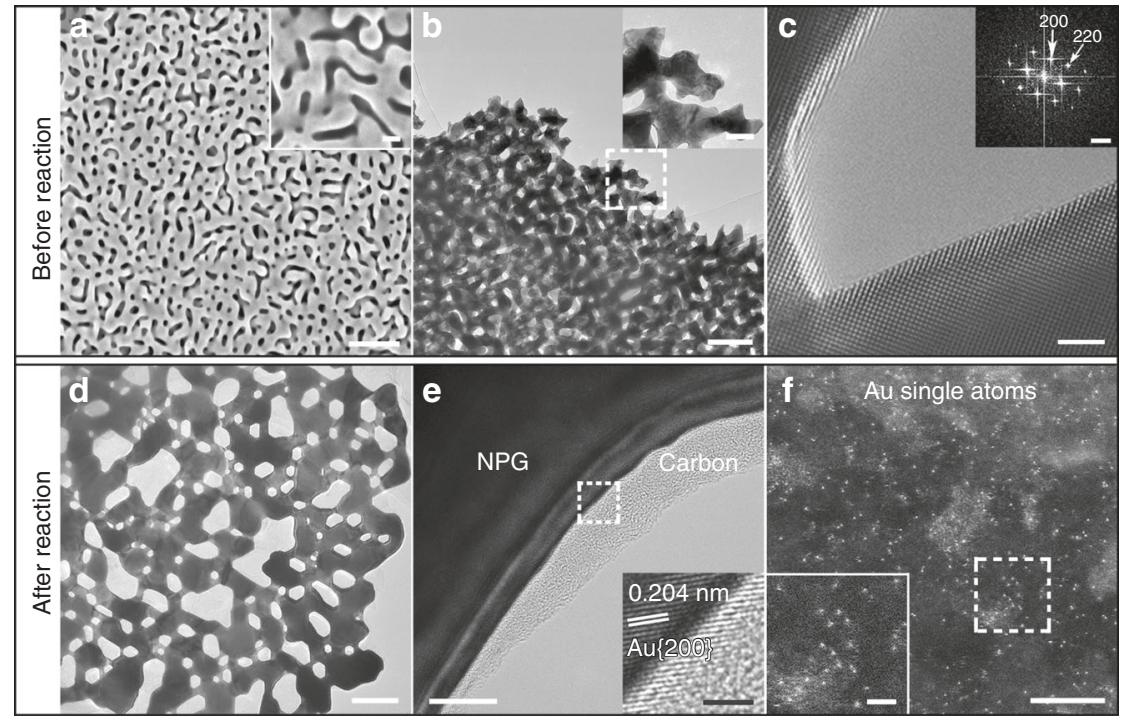

Fig. 1 Characterization of NPG before and after the ex situ catalytic $\mathbf{C H}_{\mathbf{4}}$ pyrolysis reaction. SEM (a) and TEM (b) images of an NPG sample before the reaction. The insets in $\mathbf{a}$ and $\mathbf{b}$ are a high-magnification SEM image and the close-up view of the boxed region in $\mathbf{b}$, respectively. $\mathbf{c} H R T E M$ image and its fast Fourier transform pattern. TEM (d) and HRTEM (e) images of an NPG sample after the reaction. $\mathbf{f}$ HAADF image of a carbon region on the NPG sample. The insets in $\mathbf{e}$ and $\mathbf{f}$ are the close-up views of the boxed regions in $\mathbf{e}$ and $\mathbf{f}$, respectively. Scale bars: $\mathbf{a} 200 \mathrm{~nm}$; the inset of $\mathbf{a} 50 \mathrm{~nm} ; \mathbf{b} 200 \mathrm{~nm}$; the inset of b $50 \mathrm{~nm}$; $2 \mathrm{~nm}$; the inset of c $5 \mathrm{~nm}^{-1} ; \mathbf{d} 200 \mathrm{~nm}$; e $10 \mathrm{~nm}$; the inset of e $2 \mathrm{~nm} ; \mathbf{f} 5 \mathrm{~nm}$; the inset of $\mathbf{f} 2 \mathrm{~nm}$. 
instance, the as-prepared sample in Fig. 1a-c has the average ligament size of $\sim 50 \mathrm{~nm}$. Its energy-dispersive X-ray spectroscopy result is displayed in Supplementary Figs. 1 and 2, indicating that it is $\mathrm{Au}$ containing $1.37 \pm 0.38 \mathrm{at} \% \mathrm{Ag}(0.38$ is the standard deviation, and the Source data are provided as a Source Data file) and the Ag distribution in it is homogeneous. The high-resolution TEM (HRTEM) image in Fig. 1c shows that the NPG sample has the standard crystal structure of $\mathrm{Au}$, and the surfaces of its ligaments are clean. After the reaction, most of the pores become larger, some ligaments slim and the others coarsen, amorphous carbon has been produced on the surfaces of all ligaments, and the ligaments are still $\mathrm{Au}$ (Fig. 1d, e). Importantly, HAADF imaging shows that a high density of heavy metal SAs appear in the amorphous carbon (Fig. 1f, Supplementary Figs. 3-8, 14 and Supplementary Notes $1-4,6)$ considering $C$ is substantially lighter than $\mathrm{Au}$ or $\mathrm{Ag}^{4,8,21-26,28,39-41}$. While no $\mathrm{Ag}$ signals were detected within the amorphous carbon (Supplementary Figs. 4 and 11), possibly due to its very low content in the original NPG sample, the existence of $\mathrm{Ag} \mathrm{SAs}$ is possible. Contrast intensity analysis in Supplementary Figs. 5-8 and Supplementary Notes 1-4 show the overwhelming majority of bright spots are Au SAs, and a few darker bright spots may be Ag SAs (Fig. 1f, Supplementary Figs. 3-6, 8, 14 and Supplementary Notes 1, 2, $4,6)$, especially considering the contrast intensity is also related to its distance from the focal plane (Supplementary Fig. 6 and Supplementary Note 2).

Dynamic process of the $\mathrm{CH}_{4}$ pyrolysis reaction on NPG. According to the results in Fig. 1, it is speculated that during the above-described $\mathrm{CH}_{4}$ pyrolysis reaction, the NPG surfaces experienced an evolution, causing the release of Au SAs from the surfaces. To check this speculation, in situ HRTEM was used to monitor the dynamic process of the $\mathrm{CH}_{4}$ pyrolysis reaction on NPG at $346^{\circ} \mathrm{C}$ (see details in "Methods"). It should be noted that TEM and STEM images of NPG are all projections of its 3D structure, in which two types of surface regions with positive and negative curvatures exist ${ }^{34,35}$. The dynamic processes on the two types were both recorded, as displayed in Fig. 2 and Supplementary Movies 1 and 2, which have been slowed down by eight times of the real rate ( 80 frames per second) for facilitating visual inspection. This rate means a high temporal resolution of $0.0125 \mathrm{~s}$, and Fig. 2 indicates that the spatial resolution of the in situ HRTEM technique is high enough to resolve the Au crystal lattices of NPG. More importantly, the in situ observations show that on both the two types of surface regions, the thicknesses of the amorphous carbon increased continuously, suggesting that the catalytic $\mathrm{CH}_{4}$ pyrolysis reaction continuously occurred on the surfaces. At the same time, the diameters of the two NPG ligaments decreased continuously, indicating that their surfaces continuously disintegrated, while the crystal structures within the residual ligaments were still Au because the HRTEM images show that their crystal lattices are the same as those of standard Au.

The diameter decrease of the slimmed NPG ligaments indicates that the extensive emergence of the Au SAs (Fig. 1f) was due to that a large number of Au atoms were released from the surfaces of the slimmed ligaments and were then dispersed as SAs in the amorphous carbon layers. Meantime, the diameter increase of the coarsened ligaments manifests that some of the Au SAs came back to some ligaments. These findings are very similar to the theoretically reported results that the adsorption and diffusion of $\mathrm{CO}$ molecules in the form of $\mathrm{Au}-\mathrm{CO}$ species on a $\mathrm{CeO}_{2}$ substrate cause Au SAs to break away from Au nanoparticles/clusters and the Au SAs can come back to the nanoparticles/clusters ${ }^{5,6}$. Thus, we performed density functional theory (DFT) calculations, and the result shows that $\mathrm{C}$ atoms in the amorphous carbon can adsorb on the surface of an Au crystal, and their interaction with the crystal surface pulls Au atoms out of the surface, leading to the formation of Au SAs (Supplementary Fig. 9).

It is well known that carbon deposits generally prevent the contact between reactants and catalyst surfaces and thus stop heterogeneous catalytic reactions ${ }^{13,15,16}$. But, the $\mathrm{CH}_{4}$ pyrolysis reaction on the surfaces of NPG still proceeded normally even

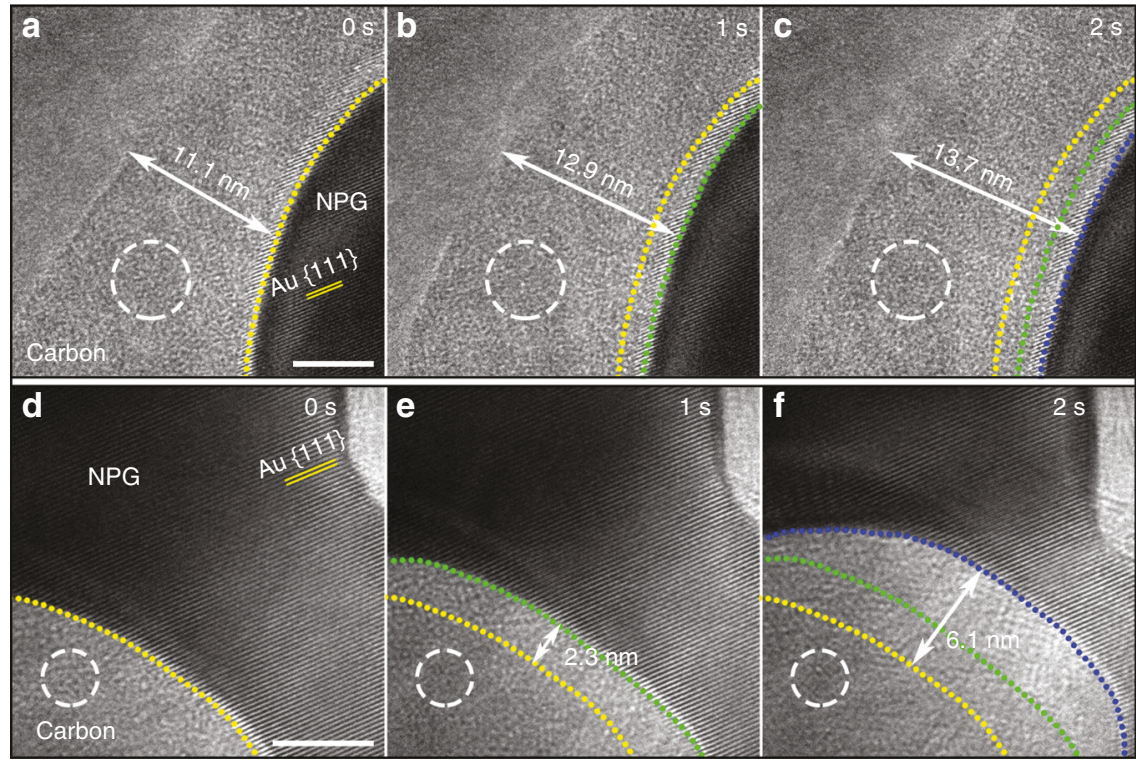

Fig. $\mathbf{2}$ In situ dynamic process of the catalytic $\mathbf{C H}_{\mathbf{4}}$ pyrolysis reaction on NPG. a-c HRTEM images at three different moments during the reaction on a surface region with positive curvature. The images have the same scale bar. The moment in $\mathbf{a}$ is defined as $0 \mathrm{~s}$. The yellow, the green, and the blue dashed lines indicate the positions of the surface at 0,1 , and $2 \mathrm{~s}$, respectively. The white dashed circles denote the positions of a randomly selected carbon region, and the arrows indicate the thickness of the amorphous carbon at 0,1 , and $2 \mathrm{~s}$. $\mathbf{d}-\mathbf{f}$ have the same meanings as a-c but are for a surface region with negative curvature. The arrows indicate the reduction of the NPG ligament at 0, 1, and $2 \mathrm{~s}$. a-c and $\mathbf{d}-\mathbf{f}$ are cut from Supplementary Movies 1 and 2 , respectively (see more details in "Methods"). Scale bars: a $5 \mathrm{~nm}$; d $5 \mathrm{~nm}$. 
when the thicknesses of the amorphous carbon layers exceeded $10 \mathrm{~nm}$ (Fig. 2 and Supplementary Movies 1 and 2). This phenomenon is due to the presence of abundant nanopores with diameters of 3-7 nm and sub-nanopores with diameters centered at $0.82 \mathrm{~nm}$ in the amorphous carbon layers (Fig. 3a and Supplementary Fig. 10), which serve as channels for the gas exchange through the amorphous carbon layers and thus effectively alleviate the poisoning effect that normally occurs on the catalyst surface. These pores should be produced by the flows of the gas-phase products $\left(\mathrm{CH}_{3}, \mathrm{CH}_{2}, \mathrm{CH}\right.$, and $\left.\mathrm{H}_{2}\right)$ during the $\mathrm{CH}_{4}$ pyrolysis, which is similar to the pore generation in gas-eutectic transformations ${ }^{42}$.

The presence of the gas channels also allows the Au SAs in the amorphous carbon layers to contact $\mathrm{CH}_{4}$ molecules and thus catalyze their pyrolysis, which is proven by the HRTEM images in Figs. 2 and 3 and Supplementary Movies 1-4. First, the images in Fig. 2 and their related movies (Supplementary Movies 1 and 2) show that the contrasts within the amorphous carbon layers including those carbon parts distant from the NPG surfaces were always dramatically changing, when the conditions of the $\mathrm{CH}_{4}$ pyrolysis reaction were kept. For instance, the carbon zone indicated by the dashed circles in Fig. 2a-c was distant from the NPG surface, but it had a changing contrast during the short period of 2 s. So did the one in Fig. 2d-f. For visual inspection, the contrast changing in the corresponding movies is clearer. In contrast, after the $\mathrm{CH}_{4}$ flow was stopped, the contrast changing became from violent to rather mild and even vanished, as shown by Fig. 3b, c and Supplementary Movie 3. For instance, the contrasts of the six zones indicated by the dashed circles in Fig. 3b, $c$ were stable and almost unchanged. The above comparison manifests that the violent changing of the contrast within the amorphous carbon parts distant from the NPG surfaces in Fig. 2 was not due to the electron beam of TEM but to a certain reaction in the carbon parts.

Further, Fig. 3d-f and its related movie (Supplementary Movie 4) show that in an amorphous carbon region distant from the NPG surface, a nanoparticle with the Au crystal structure temporarily formed and then quickly disappeared before $0.4125 \mathrm{~s}$ during the $\mathrm{CH}_{4}$ pyrolysis. Meantime, the contrast within the carbon region was also changing, such as the contrast of the zone indicated by the dashed circles in Fig. 3d-f (see Supplementary Movie 4 for clearer visual inspection). These phenomena were easily found in other amorphous carbon regions (Supplementary Figs. 11, 12 and Supplementary Movie 5). Due to the imaging mechanism of HRTEM that is different from that of HAADF ${ }^{43}$, Au SAs cannot be imaged by the HRTEM images in Figs. 2 and 3 and their related movies. Nevertheless, if no sources of $\mathrm{Au}$ atoms had existed in the amorphous carbon layers, the Au nanoparticles would not have formed. Moreover, we have randomly selected 20 different regions of amorphous carbon for HAADF imaging and found that all of them contain Au SAs. Besides, as analyzed before, the changing of the contrasts within the amorphous carbon regions suggest a certain reaction occurring in the carbon parts. This reaction has to be the $\mathrm{CH}_{4}$ pyrolysis catalyzed by $\mathrm{Au}$ SAs, because no other reactions can exist. Therefore, the above analyses imply the following. The Au SAs and NPG co-catalyzed the $\mathrm{CH}_{4}$ pyrolysis reaction. At the same time, in amorphous carbon regions distant from NPG surfaces, some Au SAs dynamically aggregated into Au nanoparticles (Fig. 3d-e, Supplementary Figs. 11-14, Supplementary Notes 5, 6 and
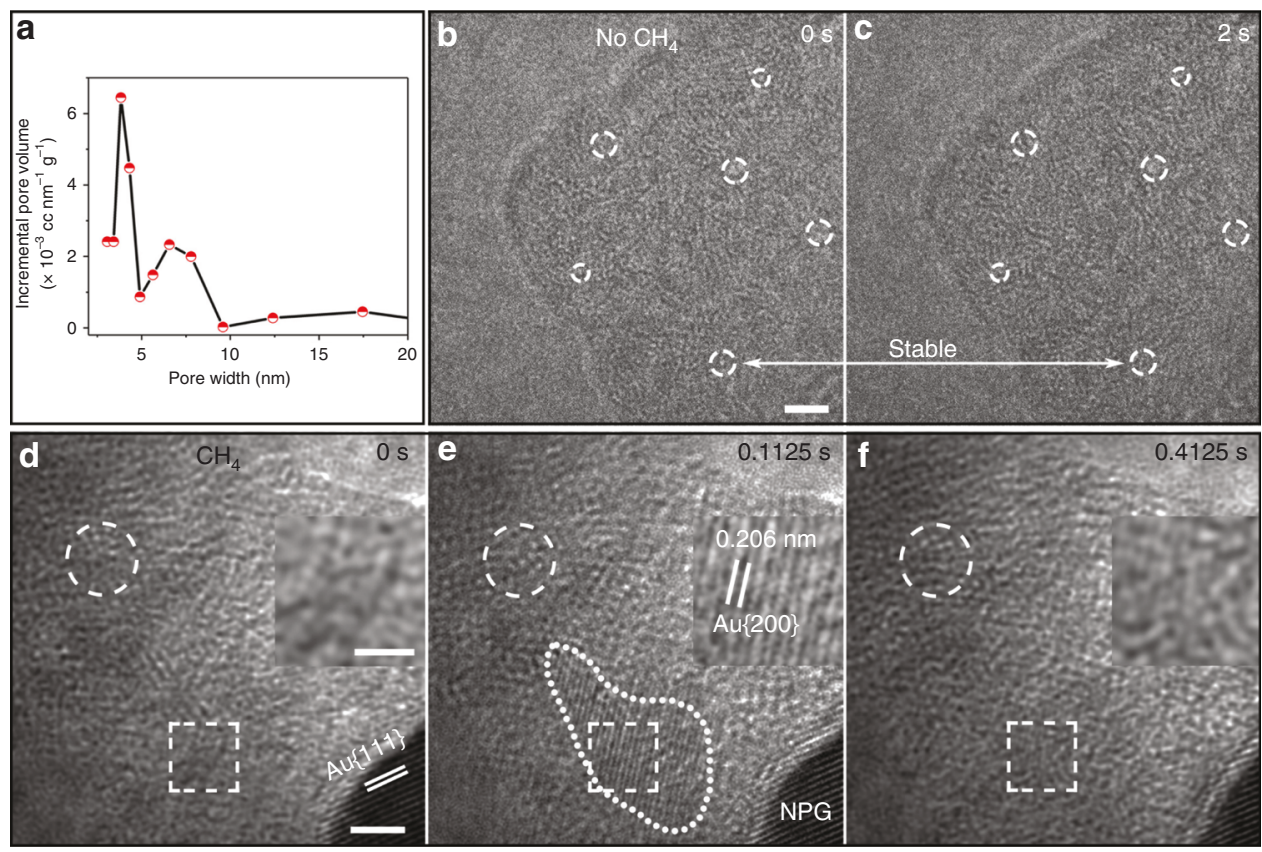

Fig. 3 Characterization of the amorphous carbon layers and in situ dynamic catalysis process of Au SAs. a Size distribution of nanopores in the amorphous carbon layers produced by the ex situ $\mathrm{CH}_{4}$ pyrolysis reaction (see more details in "Methods" and Supplementary Fig. 10). b, c HRTEM images of an amorphous carbon layer at two different moments after the layer was produced and the $\mathrm{CH}_{4}$ flow was stopped with the other conditions of the in situ $\mathrm{CH}_{4}$ pyrolysis reaction unchanged. The two images have the same scale bar. d-f HRTEM images of an amorphous carbon layer at three different moments during the reaction with all the conditions unchanged. The moments in $\mathbf{b}$ and $\mathbf{d}$ are defined as $0 \mathrm{~s}$. The white dashed circles in $\mathbf{b}$ and $\mathbf{c}$ denote the positions of six randomly selected carbon zones at 0 and $2 \mathrm{~s}$, which are typical unchanged regions, and the ones in $\mathbf{d}-\mathbf{f}$ indicate the positions of a randomly selected carbon zone at $0,0.1125$, and $0.4125 \mathrm{~s}$, which is a typical changed region. The boxes in $\mathbf{d}-\mathbf{f}$ indicate the positions of a nanoparticle that temporarily formed and disappeared. The insets in $\mathbf{d}-\mathbf{f}$ are the close-up views of the boxed regions in $\mathbf{d}-\mathbf{f}$, respectively. The dashed closed curve in $\mathbf{e}$ indicates the boundary of a temporarily formed crystal nanoparticle. b, c and $\mathbf{d}-\mathbf{f}$ are cut from Supplementary Movies 3 and 4, respectively (see more details in Methods). Scale bars: $\mathbf{b}$ $2 \mathrm{~nm}$; $2 \mathrm{~nm}$; the inset of $\mathbf{d} 1 \mathrm{~nm}$. 
Supplementary Movies 4, 5), which re-disintegrated back to SAs (Fig. 3e-f, Supplementary Figs. 12, 13, Supplementary Note 5 and Supplementary Movies 4-6), similar to the catalysis-induced surface disintegration and ligament coarsening in Figs. $1 \mathrm{~d}$ and 2. This phenomenon suggests that the Au SAs and the nanoparticles also co-catalyzed the $\mathrm{CH}_{4}$ pyrolysis reaction, which is confirmed by DFT calculations below.

\section{Discussion}

The catalytic reactivity of Au SAs toward the $\mathrm{CH}_{4}$ pyrolysis was checked by performing DFT calculations on a model of an Au SA anchored on an amorphous carbon substrate. The DFT result is shown in Supplementary Fig. 15, indicating that a $\mathrm{CH}_{4}$ molecule is firstly adsorbed on a $\mathrm{C}$ atom of the substrate by a weak interaction. Then, the four $\mathrm{H}$ atoms are gradually removed to form the final product $\mathrm{C}$ through the interactions between the intermediates, the Au SA and the substrate. The DFT result also manifests that the above process is feasible under the actual reaction conditions.

The experimental aggregation of Au SAs into Au nanoparticles during the catalytic $\mathrm{CH}_{4}$ pyrolysis is easily understandable because $\mathrm{Au}$ SAs have much higher surface energies than their counterpart nanoparticles/clusters ${ }^{23-25,28}$. To gain insight into the re-disintegration of the temporarily formed Au nanoparticles, we have also performed DFT calculations. Figure 4 displays the calculated results. Initially, an Au cluster is loaded on amorphous carbon (Fig. 4a). Figure 4b, c shows that when the intermediate $\mathrm{CH}_{3}{ }^{*}$ of the $\mathrm{CH}_{4}$ pyrolysis is adsorbed on the Au cluster, the distances between the $\mathrm{Au}$ atom with $\mathrm{CH}_{3}{ }^{*}$ adsorbed and the neighbors in the cluster become from 3.42 and $2.78 \AA$ to 4.52 and $4.67 \AA$. That is, the adsorption of $\mathrm{CH}_{3}{ }^{*}$ weakens the interaction between the $\mathrm{Au}$ atom and the cluster, and thus this $\mathrm{Au}$ atom is inclined to break away from the cluster. Similar results are also found in $\mathrm{CH}_{2}-\mathrm{Au} / \mathrm{C}, \mathrm{CH}-\mathrm{Au} / \mathrm{C}$, and $\mathrm{C}-\mathrm{Au} / \mathrm{C}$ (Supplementary Fig. 16). The energy change from an initial state (IS) to its corresponding transition state (TS) is the activation energy $\left(E_{\mathrm{a}}\right)$. Figure $4 \mathrm{~d}$ gives the $E_{\mathrm{a}}$ values of the configuration evolutions of $\mathrm{CH}_{3}-\mathrm{Au} / \mathrm{C}, \mathrm{CH}_{2}-\mathrm{Au} / \mathrm{C}, \mathrm{CH}-\mathrm{Au} / \mathrm{C}$ and $\mathrm{C}-\mathrm{Au} / \mathrm{C}$ to be $0.77,0.01$, 0.31 , and $0.47 \mathrm{eV}$, respectively. These values are all easily achievable under the actual reaction conditions, such as the high temperatures of 580 and $346^{\circ} \mathrm{C}^{44}$. That is, all of the final states (FSs) in Fig. 4b-d can be easily realized under the actual reaction conditions, indicating not only the efficient catalytic reactivity of the $\mathrm{Au}$ cluster toward the $\mathrm{CH}_{4}$ pyrolysis but also the feasibility of the re-disintegration of the temporarily formed $\mathrm{Au}$ nanoparticles into $\mathrm{Au}$ SAs. Moreover, we have also calculated $\mathrm{Au}$ clusters composed of 3 and 2 atoms (namely $\mathrm{Au}_{3}$ and $\mathrm{Au}_{2}$ ) and found that before the clusters can catalyze the conversion of $\mathrm{CH}_{3}{ }^{*}$ into $\mathrm{CH}_{2}{ }^{*}$, they should have broken into three or two Au SAs (see more details in Supplementary Figs. 17 and 18).

In addition to the dynamic processes of $\mathrm{Au}$ SAs and NPG ligaments we observed and calculated above, in situ TEM studies of $\mathrm{CO}$ oxidation on $\mathrm{NPG}^{34,38}$ revealed dramatically enhanced surface diffusion of $\mathrm{Au}$ which led to substantial coarsening of some pores and some Au ligaments. In our work, the coarsening also occurs during the $\mathrm{CH}_{4}$ pyrolysis, as indicated by Fig. $1 \mathrm{~b}, \mathrm{~d}$. Our in situ observation shows that the breaking and migration of a ligament can make its adjacent ligament coarsen (see details in Supplementary Fig. 19).

It should be noted that the incident high-energy $(200 \mathrm{keV})$ electrons might influence the in situ TEM experiments. To explore this, we performed continuous electron-beam irradiation experiments on NPG at $23^{\circ} \mathrm{C}$ and during heating without and with $\mathrm{CH}_{4}$, and an on-line mass spectrometer was used to detect hydrogen production when the electron beam was turned off (see details in Methods and Supplementary Figs. 19-25, Supplementary Note 7). The results clearly indicate that not the electron beam but the NPG-catalyzed $\mathrm{CH}_{4}$ pyrolysis plays the dominant role in the NPG structure evolution and the migration of $\mathrm{Au}$ atoms from the NPG surfaces (see more details in Supplementary Figs. 23-25 and Supplementary Note 7). This conforms to a published theoretical result ${ }^{45}$, which indicates that the minimum incident-electron energy to knock out $\mathrm{Au}$ atoms is approximately $407 \mathrm{keV}$, much higher than $200 \mathrm{keV}$ of the electron irradiation used in our work.

It should be noted that the recorded hydrogen production profiles on NPG samples with different Ag contents indicate that the residual Ag may contribute to the catalytic $\mathrm{CH}_{4}$ pyrolysis, especially at temperatures below $200{ }^{\circ} \mathrm{C}$ (see more details in Supplementary Figs. 11, 26 and Supplementary Note 8). In order to evaluate the role of the residual Ag of NPG in the $\mathrm{CH}_{4}$ pyrolysis, we fabricated a series of NPG samples with different Ag contents and detected their hydrogen production (see details in Methods and Supplementary Fig. 26, Supplementary Note 8). The results show that with the decrease of the Ag content, the lowtemperature $\left(<200^{\circ} \mathrm{C}\right)$ activity decreases remarkably, and for our discussed NPG sample with the lowest Ag content of $1.37 \pm 0.38$
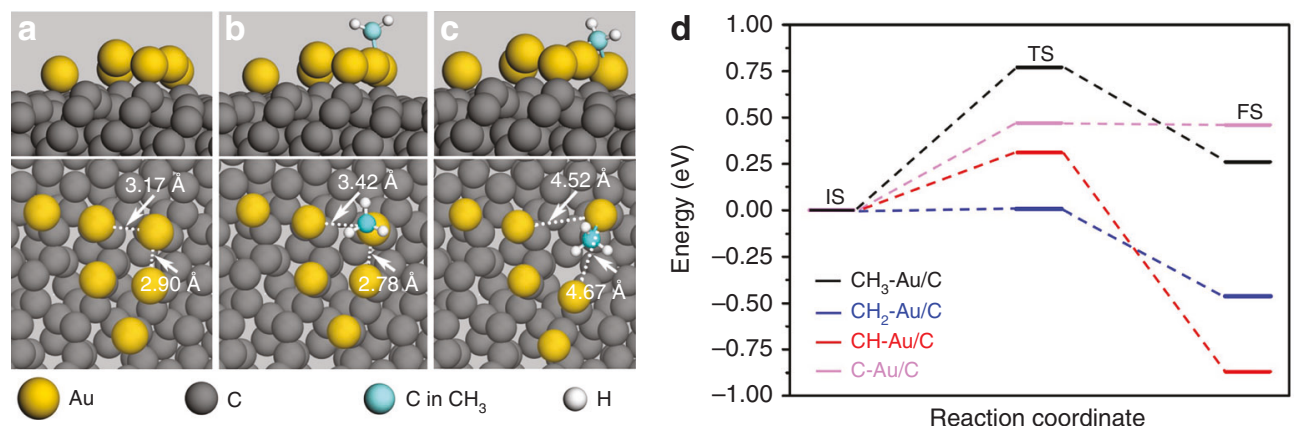

Fig. 4 DFT-calculated configuration evolutions of an Au cluster on an amorphous carbon. a DFT-relaxed atomic configuration of a pure Au cluster consisting of six Au atoms on amorphous carbon, which is denoted as Au/C. The top and the bottom panels are the side and the top views of the configuration, respectively. b, c DFT-relaxed atomic configurations of the IS (b) and the FS (c) of $\mathrm{CH}_{3}-\mathrm{Au} / \mathrm{C}$ (see Supplementary Fig. 16 for the configurations of $\mathrm{CH}_{2}-\mathrm{Au} / \mathrm{C}, \mathrm{CH}-\mathrm{Au} / \mathrm{C}$ and $\left.\mathrm{C}-\mathrm{Au} / \mathrm{C}\right) . \mathrm{CH}_{3}-\mathrm{Au} / \mathrm{C}, \mathrm{CH}_{2}-\mathrm{Au} / \mathrm{C}, \mathrm{CH}-\mathrm{Au} / \mathrm{C}$ and $\mathrm{C}-\mathrm{Au} / \mathrm{C}$ denote the configurations of the $\mathrm{Au}$ cluster, respectively, with the presence of $\mathrm{CH}_{3}{ }^{*}, \mathrm{CH}_{2}{ }^{*}, \mathrm{CH}^{\star}$ and $\mathrm{C}^{*}$, which are the intermediates and the carbon product of the $\mathrm{CH}_{4}$ pyrolysis. IS, TS and $\mathrm{FS}$ are the initial, the transition and the final states, respectively. The dashed lines pointed by the white arrows in a-c denote the interatomic distances. $\mathbf{d}$ Activation energies of the configuration evolutions of the Au cluster, in which the black, blue, red, and pink lines represent the corresponding IS, TS, FS and activation energy of the $\mathrm{Au}$ cluster evolution under the adsorption of $\mathrm{CH}_{3}, \mathrm{CH}_{2}, \mathrm{CH}$, and $\mathrm{C}$, respectively. 
at $\%$, it becomes active only when the reaction temperature reaches $200^{\circ} \mathrm{C}$, distinct from other samples with higher $\mathrm{Ag}$ contents. Considering the catalytic activity of NPG itself has a significant structural dependence ${ }^{34-36,38}$, we cannot simply infer from the Supplementary Fig. 26 and Supplementary Note 8 whether there is a linear relationship between the Ag content and its catalytic activity for methane pyrolysis.

Actually, we selected this particular sample (with such a low Ag content) because previous report ${ }^{14}$ and our experiment (see details in Methods and Supplementary Fig. 27) prove that $\mathrm{CH}_{4}$ can be pyrolyzed by the catalysis of pure gold, and the drastic structural change of this particular sample (with such a low Ag content) provides clear evidence of how Au ligaments restructure and how $\mathrm{Au}$ SAs form, aggregate and re-disintegrate (Figs. 2 and 3 and Supplementary Movies 1, 2, 4 and 5). It is quite unlikely for such low Ag content to cause such drastic change processes, and no Ag signals were detected in the carbon layers (Supplementary Figs. 4 and 11). On the other hand, a series of transition metal clusters have been studied ${ }^{46,47}$ for their abilities to catalyze the pyrolysis of methane, and the results show that the overall ability of $\mathrm{Ag}$ to activate $\mathrm{C}-\mathrm{H}$ bonds of methane is actually slightly lower than that of $\mathrm{Au}$ ( $\mathrm{Ag}$ is only slightly better than $\mathrm{Au}$ in activating the fourth $\mathrm{C}-\mathrm{H}$ bond of methane ${ }^{48}$ (Supplementary Table 1). Considering the possible differences in actual structures of the catalysts (Au, Ag, AuAg alloys, nanoparticles, or NPG with different size), it is thus reasonable to propose that the ability of Ag to catalyze the methane pyrolysis would not be substantially stronger than that of $\mathrm{Au}$. This is the reason to speculate that in the case of extremely low Ag content, the drastic dynamic catalytic behavior of the NPG catalyst should be dominated by Au.

In summary, NPG is discovered to release a large number of $\mathrm{Au}$ SAs during its catalysis toward the methane pyrolysis. The dynamic process of this heterogeneous catalysis is directly observed by in situ HRTEM with high spatial and temporal resolutions, indicating that the NPG surfaces partially disintegrate. This disintegration is continuous, by which Au SAs are produced. As demonstrated by DFT calculation, the Au SAs can co-catalyze the reaction with nanoporous Au. At the same time, some of the SAs dynamically aggregate into Au nanoparticles, which temporarily exist and dynamically re-disintegrate into $\mathrm{Au}$ SAs. The Au SAs and the nanoparticles also co-catalyze the methane pyrolysis. These findings are confirmed by DFT calculations. This work manifests that the heterogeneous catalysis processes of nanocatalysts and SACs may need to be investigated integratedly, and the designs of nanocatalysts and SACs may not be independent from each other.

\section{Methods}

Sample preparations. The NPG samples were synthesized by dealloying 12 -karat $\mathrm{Au}-\mathrm{Ag}$ alloy films with a thickness of $100 \mathrm{~nm}$ in concentrated $\mathrm{HNO}_{3}$ at $30{ }^{\circ} \mathrm{C}$ for $30 \mathrm{~min}$. Then, the dealloyed films were immersed in ultrapure water for several times to clean them. Finally, the films floated on the surface of ultrapure water, and they were NPG pieces. This synthesis is the same as reported ${ }^{34-38}$. For conventional and in situ TEM characterizations, conventional TEM grids and sample chips from the DENS solutions company at The Netherlands were used, respectively, to collect NPG pieces from the water surface. In particular, for the in situ study, no plasma was used to treat NPG pieces before they were inserted into TEM. For other characterizations and the ex situ $\mathrm{CH}_{4}$ pyrolysis, some NPG pieces were dried under an infrared lamp. In addition, we used a magnetron sputtering instrument (JGP-450C, SKY Technology Development Co., Ltd, Chinese Academy of Sciences) with a pure $\mathrm{Au}(99.99 \%)$ and $\mathrm{Au}_{1} \mathrm{Ag}_{1}$ (99.99\%) target to produce pure $\mathrm{Au}$ nanoparticles as a control sample and $\mathrm{Au}$ and $\mathrm{Ag}$ SAs co-existing on ultra-thin carbon films (Beijing XXBR Technology Co., Ltd).

Ex situ $\mathbf{C H}_{\mathbf{4}}$ pyrolysis. The pyrolysis was performed in a dc-PECVD system (Black Magic 6-inch, AIXTRON). An NPG sample was loaded into the reaction chamber and was heated to $580^{\circ} \mathrm{C}$ under $200 \mathrm{sccm} \mathrm{Ar}$ and $20 \mathrm{sccm} \mathrm{H}$ with a chamber pressure of $5 \mathrm{mbar}$. Then, the plasma ( $80 \mathrm{~W}$ power) was turned on, and $50 \mathrm{sccm}$ $\mathrm{CH}_{4}$ was introduced into the chamber for 1-2 h. Afterwards, the plasma and the
$\mathrm{CH}_{4}$ flow were stopped. This experiment can rapidly produce a mass of carbonloaded NPG with SAs, whose structures are shown in Fig. 1d, $\mathrm{f}$ and quite similar to those of the samples through the in situ study (Supplementary Figs. 28 and 29).

Pore size distribution. After the ex situ $\mathrm{CH}_{4}$ pyrolysis, $100 \mathrm{mg}$ NPG with amorphous carbon was used to evaluate the pore structure of the amorphous carbon, which was performed using $\mathrm{N}_{2}(77 \mathrm{~K})$ and $\mathrm{CO}_{2}(273 \mathrm{~K})$ adsorption-desorption measurements on an Autosorb-IQ-MP Micromeritics analyzer. Pore volumes were computed by the Barrett-Joyner-Halenda (BJH) method, the size distribution of nanopores was determined by the Brunauer-Emmett-Teller (BET) method by using the $\mathrm{N}_{2}$ adsorption branch under $77 \mathrm{~K}$, and the size distribution of sub-nanopores was determined by the nonlocal density functional theory model based on the $\mathrm{CO}_{2}$ adsorption branch at $273 \mathrm{~K}$.

TEM experiments. The conventional TEM characterizations were performed with three TEM instruments of Talos F200X, Titan Cubed Themis G2 300 and Themis $\mathrm{Z}$ from FEI at $200 \mathrm{kV}$. For each of the in situ TEM experiments, a DENSsolutions chip with NPG pieces was assembled into an in situ TEM gas-phase holder (Climate $\mathrm{S} 3+$, DENSsolutions), whose specified range of error for temperature is $<5 \%$. The holder was then inserted into Talos F200X with a high-speed camera (Ceta 2, FEI) to record in situ TEM movies/images at $200 \mathrm{kV}$, and the dose rate of the electron beam applied for the in situ experiments was $0.039-1570 \mathrm{e}^{-2} \mathrm{~s}^{-1}$, which was adjusted by the magnification $(\times 4300-\times 1,050,000)$. Before the in situ catalytic reaction, the gas line of the holder was flushed with Ar for $30 \mathrm{~min}$ at atmospheric pressure, and $\mathrm{CH}_{4}$ was then introduced. Afterwards, the temperature was increased by $30^{\circ} \mathrm{C} \mathrm{min}-1$ to $346^{\circ} \mathrm{C}$, at which we found amorphous carbon to start to grow significantly and then recorded movies with the HRTEM mode and the camera. The gases of pure $\mathrm{CH}_{4}$ (99.995\%) and $\mathrm{Ar}$ (99.999\%) were purchased from Beijing ZG Special Gases Science \& Technology Co., Ltd (Beijing, China). The on-line mass spectrometer used to detect hydrogen production is Omnistar GSD $320 \mathrm{O} 1$ (Pfeiffer Vacuum, Germany). For facilitating visual inspection, Supplementary Movies 1-5 have been slowed down by eight times of the real rate ( 80 frames per second), in which the moments corresponding to the images in Figs. 2 and $3 b-f$ and Supplementary Fig. 12 are paused for a short while. The HRTEM images in Figs. 2 and $3 \mathrm{~d}-\mathrm{f}$ and Supplementary Fig. 12 are Wiener-filtered, and the Wiener filtering has been widely used to remove noise in HRTEM and high-resolution STEM images ${ }^{27,49}$.

DFT calculations. The calculations were performed using the generalized gradient approximation-Perdew, Burke, and Ernzerh of (PBE) functional ${ }^{50}$ as implemented in the all-electron $\mathrm{DMol} 3 \operatorname{code}^{51,52}$. The double numerical plus polarization basis set was used throughout the calculations. The convergence criteria were set to be $2 \times 10^{-5} \mathrm{Ha}, 0.004 \mathrm{Ha}^{-1}$ and $0.005 \AA$ for the energy, the force and the displacement convergences, respectively. A self-consistent field density convergence with a threshold value of $1 \times 10^{-5} \mathrm{Ha}$ was specified. In the computation of the formation of Au SAs from NPG surfaces, $3 \times 3$ Au $\{311\}$ cells with the thickness of six atomic layers were used to represent the NPG surface. In the computation of the methane pyrolysis reaction mechanism, an amorphous carbon layer of $8.65 \AA \times 9.98 \AA \times 6 \AA$ was selected as the carbon carrier, on which an Au SA was located. In the computation of the configuration evolutions of the Au cluster, an amorphous carbon layer of $14.5 \AA \times 15.0 \AA \times 4 \AA$ was selected to represent the amorphous carbon carrier, on which six Au atoms were placed to simulate the Au cluster. In all cases, a vacuum region of $30 \AA$ was used to ensure negligible interaction between periodic replicas. All the TSs were determined by the linear synchronous transit and quadratic synchronous transit methods. The TS structures were characterized by analyzing the vibrational normal modes and by using a local minimum search (after a small distortion of each TS in the reaction coordinate direction) to reach the reactants and products ${ }^{53}$.

\section{Data availability}

The data that support the findings of this study are available within the article (and its Supplementary Information files) and from the corresponding authors upon reasonable request. The source data underlying the $\mathrm{Ag}$ content of $1.37 \pm 0.38$ at $\%$ are provided as a Source Data file.

Received: 1 February 2019; Accepted: 23 March 2020; Published online: 21 April 2020

\section{References}

1. Yao, S. et al. Atomic-layered Au clusters on $\mathrm{a}-\mathrm{MoC}$ as catalysts for the lowtemperature water-gas shift reaction. Science 357, 389-393 (2017).

2. Li, S. et al. Tuning the selectivity of catalytic carbon dioxide hydrogenation over iridium/cerium oxide catalysts with a strong metal-support interaction. Angew. Chem. Int. Ed. 56, 10761-10765 (2017). 
3. Zhang, X. et al. Highly dispersed copper over $\beta-\mathrm{Mo}_{2} \mathrm{C}$ as an efficient and stable catalyst for the reverse water gas shift (RWGS) reaction. ACS Catal. 7, 912-918 (2017).

4. Qiao, B. et al. Single-atom catalysis of $\mathrm{CO}$ oxidation using $\mathrm{Pt}_{1} / \mathrm{FeO}_{\mathrm{x}}$. Nat. Chem. 3, 634-641 (2011).

5. Wang, Y. G., Mei, D., Glezakou, V. A., Li, J. \& Rousseau, R. Dynamic formation of single-atom catalytic active sites on ceria-supported gold nanoparticles. Nat. Commun. 6, 6511 (2015).

6. Liu, J. C., Wang, Y. G. \& Li, J. Toward rational design of oxide-supported single-atom catalysts: atomic dispersion of gold on ceria. J. Am. Chem. Soc. 139, 6190-6199 (2017).

7. Lin, J. et al. Design of a highly active $\mathrm{Ir} / \mathrm{Fe}(\mathrm{OH})_{\mathrm{x}}$ catalyst: versatile application of Pt-group metals for the preferential oxidation of carbon monoxide. Angew. Chem. Int. Ed. 124, 2974-2978 (2012).

8. Qiao, B. et al. Highly efficient catalysis of preferential oxidation of $\mathrm{CO}$ in $\mathrm{H}_{2}$ rich stream by gold single-atom catalysts. ACS Catal. 5, 6249-6254 (2015).

9. Zhong, D. et al. Linear alkane polymerization on a gold surface. Science 334, 213-216 (2011).

10. Agarwal, N. et al. Aqueous Au-Pd colloids catalyze selective $\mathrm{CH}_{4}$ oxidation to $\mathrm{CH}_{3} \mathrm{OH}$ with $\mathrm{O}_{2}$ under mild conditions. Science 358, 223-226 (2017).

11. Chen, D. et al. Synthesis of carbon nanofibers: effects of Ni crystal size during methane decomposition. J. Catal. 229, 82-96 (2005).

12. Du, X., Zou, G., Zhang, Y. \& Wang, X. A novel strategy for low-temperature synthesis of ruddlesden-popper type layered perovskite $\mathrm{La}_{3} \mathrm{Mn}_{2} \mathrm{O}_{7+\delta}$ for methane combustion. J. Mater. Chem. A 1, 8411-8416 (2013).

13. $\mathrm{Xu}, \mathrm{S}$. \& Wang, $\mathrm{X}$. Highly active and coking resistant $\mathrm{Ni} / \mathrm{CeO}_{2}-\mathrm{ZrO}_{2}$ catalyst for partial oxidation of methane. Fuel 84, 563-567 (2005).

14. Lang, S. M., Bernhardt, T. M., Chernyy, V., Bakker, J. M., Barnett, R. N. \& Landman, U. Selective C-H bond cleavage in methane by small gold clusters. Angew. Chem. Int. Ed. 56, 13406-13410 (2017).

15. Ashik, U. P. M., Daud, W. M. A. W. \& Abbas, H. F. Production of greenhouse gas free hydrogen by thermocatalytic decomposition of methane-a review. Renew. Sustain. Energy Rev. 44, 221-256 (2015).

16. Zhang, J. et al. Hydrogen production by catalytic methane decomposition: carbon materials as catalysts or catalyst supports. Int. J. Hydrog. Energy 42 19755-19775 (2017).

17. Li, Z. et al. Platinum-nickel frame within metal-organic framework fabricated in situ for hydrogen enrichment and molecular sieving. Nat. Commun. 6, 8248 (2015).

18. Li, W. Z. et al. Chemical insights into the design and development of facecentered cubic ruthenium catalysts for fischer-tropsch synthesis. J. Am. Chem. Soc. 139, 2267-2276 (2017).

19. Ji, N. et al. Direct catalytic conversion of cellulose into ethylene glycol using nickel-promoted tungsten carbide catalysts. Angew. Chem. Int. Ed. 47, 8510-8513 (2008).

20. Wan, D. et al. Low-temperature aluminum reduction of graphene oxide, electrical properties, surface wettability, and energy storage applications. ACS Nano 6, 9068-9078 (2012).

21. Lin, L. et al. Low-temperature hydrogen production from water and methanol using Pt/a-MoC catalysts. Nature 544, 80-83 (2017).

22. Lang, R. et al. Non defect-stabilized thermally stable single-atomcatalyst. Nat. Commun. 10, 234 (2019).

23. Wan, J. et al. Defect effects on $\mathrm{TiO}_{2}$ nanosheets: stabilizing single atomic site $\mathrm{Au}$ and promoting catalytic properties. Adv. Mater. 30, 1705369 (2018).

24. Wang, A., Li, J. \& Zhang, T. Heterogeneous single-atom catalysis. Nat. Rev. Chem. 2, 65-81 (2018)

25. Yang, X. F., Wang, A., Qiao, B., Li, J., Liu, J. \& Zhang, T. Single-atom catalysts: a new frontier in heterogeneous catalysis. Acc. Chem. Res. 46, 1740-1748 (2013).

26. $\mathrm{Li}, \mathrm{T}$. et al. Maximizing the number of interfacial sites in single-atom catalysts for the highly selective, solvent-free oxidation of primary alcohols. Angew. Chem. Int. Ed. 57, 7795-7799 (2018).

27. Zhao, J., Deng, Q., Avdoshenko, S. M., Fu, L., Eckert, J. \& Rümmeli, M. H. Direct in situ observations of single Fe atom catalytic processes and anomalous diffusion at graphene edges. Proc. Natl Acad. Sci. USA 111, 15641-15646 (2014)

28. Bayatsarmadi, B., Zheng, Y., Vasileff, A. \& Qiao, S. Z. Recent advances in atomic metal doping of carbon-based nanomaterials for energy conversion. Small 13, 1700191 (2017).

29. Patera, L. L. et al. Real-time imaging of adatom-promoted graphene growth on nickel. Science 359, 1243-1246 (2018).

30. Cao, K. et al. Comparison of atomic scale dynamics for the middle and late transition metal nanocatalysts. Nat. Commun. 9, 3382 (2018)

31. Cao, K., Chamberlain, T. W., Biskupek, J., Zoberbier, T., Kaiser, U. \& Khlobystov, A. N. Direct correlation of carbon nanotube nucleation and growth with the atomic structure of Rhenium nanocatalysts stimulated and imaged by the electron beam. Nano Lett. 18, 6334-6339 (2018).
32. Wittstock, A., Zielasek, V., Biener, J., Friend, C. M. \& Bäumer, M. Nanoporous gold catalysts for selective gas-phase oxidative coupling of methanol at low temperature. Science 327, 319-322 (2010).

33. Sun, S., Chen, X., Badwe, N. \& Sieradzki, K. Potential-dependent dynamic fracture of nanoporous gold. Nat. Mater. 14, 894-898 (2015).

34. Fujita, T. et al. Atomic origins of the high catalytic activity of nanoporous gold. Nat. Mater. 11, 775-780 (2012)

35. Liu, P. et al. Visualizing under-coordinated surface atoms on $3 \mathrm{D}$ nanoporous gold catalysts. Adv. Mater. 28, 1753-1759 (2016).

36. Zhang, X. \& Ding, Y. Unsupported nanoporous gold for heterogeneous catalysis. Catal. Sci. Technol. 3, 2862-2868 (2013).

37. Kamiuchi, N. et al. Self-activated surface dynamics in gold catalysts under reaction environments. Nat. Commun. 9, 2060 (2018).

38. Fujita, T. et al. Atomic observation of catalysis-induced nanopore coarsening of nanoporous gold. Nano Lett. 14, 1172-1177 (2014).

39. Zhang, C. L. et al. Electrospun metal-organic framework nanoparticle fibers and their derived electrocatalysts for oxygen reduction reaction. Nano Energy 55, 226-233 (2019).

40. Zhang, L., Han, L., Liu, H., Liu, X. \& Luo, J. Potential-cycling synthesis of single platinum atoms for efficient hydrogen evolution in neutral media. Angew. Chem. Int. Ed. 56, 13694-13698 (2017).

41. Han, L. et al. Atomically dispersed molybdenum catalysts for efficient ambient nitrogen fixation. Angew. Chem. Int. Ed. 58, 2321-2325 (2019).

42. Karpov, V. Y. Properties of gasars-metallic materials with pores formed by released hydrogen. Mater. Sci. 43, 746-749 (2007).

43. Williams, D. B. \& Carter, C. B. Transmission Electron Microscopy: a Textbook for Materials Science (Springer, New York, 2009).

44. Young, D. C. Computational Chemistry: A Practical Guide for Applying Techniques to Real-world Problems (A John Wiley \& Sons Publication, New York, 2001).

45. Egerton, R. F., McLeod, R., Wang, F. \& Malac, M. Basic questions related to electron-induced sputtering in the TEM. Ultramicroscopy 110, 991-997 (2010).

46. Au, C. T., Ng, C. F. \& Liao, M. S. Methane dissociation and syngas formation on $\mathrm{Ru}, \mathrm{Os}, \mathrm{Rh}, \mathrm{Ir}, \mathrm{Pd}, \mathrm{Pt}, \mathrm{Cu}, \mathrm{Ag}$, and $\mathrm{Au}$ : a theoretical study. J. Catal. 185, 12-22 (1999).

47. Liao, M. S. \& Zhang, Q. E. Dissociation of methane on different transition metals. J. Mol. Catal. A Chem. 136, 185-194 (1998).

48. Kurnaz, E., Fellah, M. F. \& Onal, I. A density functional theory study of $\mathrm{C}-\mathrm{H}$ bond activation of methane on a bridge site of M-O-M-ZSM-5 Clusters $(\mathrm{M}=\mathrm{Au}, \mathrm{Ag}, \mathrm{Fe}$ and $\mathrm{Cu})$. Microporous. Mesoporous. Mater. 138, 68-74 (2011).

49. $\mathrm{Li}$, J. et al. Surface evolution of a Pt-Pd-Au electrocatalyst for stable oxygen reduction. Nat. Energy 2, 17111 (2017).

50. Perdew, J. P., Burke, K. \& Ernzerhof, M. Generalized gradient approximation made simple. Phys. Rev. Lett. 77, 3865-3868 (1996).

51. Delley, B. An all-electron numerical method for solving the local density functional for polyatomic molecules. J. Chem. Phys. 92, 508-517 (1990).

52. Delley, B. From molecules to solids with the DMol3 approach. J. Chem. Phys. 113, 7756-7764 (2000).

53. Frash, M. V., Kazansky, V. B., Rigby, A. M. \& Van Santen, R. A. Cracking of hydrocarbons on zeolite catalysts: density functional and hartree-fock calculations on the mechanism of the $\beta$-scission reaction. J. Phys. Chem. B 102, 2232-2238 (1998).

\section{Acknowledgements}

This work was financially supported by National Key R\&D Program of China (2017YFA0700104), National Science Fund for Distinguished Young Scholars (51825102), National Natural Science Foundation of China (51671145, 21506148, 51761165012, and 51971157), Tianjin Science Fund for Distinguished Young Scholars (19JCJQJC61800), and National Program for Thousand Young Talents of China. We acknowledge the National Supercomputing Center in Shenzhen for providing the computational resources and materials studio (version 7.0, DMol3). We thank $\mathrm{Mr}$ Zhaolong Chen at Peking University for assistance with the PECVD system and Professor Botao Qiao at Dalian Institute of Chemical Physics for useful discussion.

\section{Author contributions}

W.X. and K.W. co-performed the TEM experiments and the $\mathrm{CH}_{4}$ pyrolysis reactions. Y.S. carried out the theoretical calculations. M.G. prepared the NPG samples. Z.D. assisted in the TEM experiments. Y.Z. performed the gas adsorption-desorption experiments and analyzed the data, to which W.X., Q.C., G.H., and J.L. contributed. Y.D G.H., and J.L. co-supervised the project. All authors contributed to the data analyses and discussion and wrote the manuscript.

\section{Competing interests}

The authors declare no competing interests. 


\section{Additional information}

Supplementary information is available for this paper at https://doi.org/10.1038/s41467020-15806-8.

Correspondence and requests for materials should be addressed to Y.D., G.H. or J.L.

Peer review information Nature Communications thanks the anonymous reviewer(s) for their contribution to the peer review of this work. Peer reviewer reports are available.

Reprints and permission information is available at http://www.nature.com/reprints

Publisher's note Springer Nature remains neutral with regard to jurisdictional claims in published maps and institutional affiliations. (c) (i) Open Access This article is licensed under a Creative Commons Attribution 4.0 International License, which permits use, sharing, adaptation, distribution and reproduction in any medium or format, as long as you give appropriate credit to the original author(s) and the source, provide a link to the Creative Commons license, and indicate if changes were made. The images or other third party material in this article are included in the article's Creative Commons license, unless indicated otherwise in a credit line to the material. If material is not included in the article's Creative Commons license and your intended use is not permitted by statutory regulation or exceeds the permitted use, you will need to obtain permission directly from the copyright holder. To view a copy of this license, visit http://creativecommons.org/ licenses/by/4.0/.

(C) The Author(s) 2020 